\title{
Differential effects of secreted frizzled-related proteins (sFRPs) on osteoblastic differentiation of mouse mesenchymal cells and apoptosis of osteoblasts
}

\author{
Sun Wook Cho, Sun Ju Her, Hyun Jin Sun, Ok Kyong Choi, Jae-Yeon Yang, Sang Wan Kim, \\ Seong Yeon Kim, Chan Soo Shin *
}

Department of Internal Medicine, Seoul National University College of Medicine, 28 Yungun-Dong, Chongno-Gu, Seoul 110-744, Republic of Korea

Received 19 December 2007

Available online 31 December 2007

\begin{abstract}
Secreted frizzled-related proteins (sFRPs) are modulators of Wnt signaling. This study was undertaken for definitive assessment of contribution of different sFRPs in osteoblastic differentiation of mesenchymal progenitor cells and apoptosis of osteoblasts. Treatment of C3H10T1/2 cells with sFRP-2 at concentrations of 10, 50, and $100 \mathrm{nM}$ and sFRP-4 at low concentrations (5 nM) significantly increased Wnt-3A-induced alkaline phosphatase (ALP) activities, whereas sFRP-1 or 3 did not. Retroviral transduction of the sFRP2 but not other sFRPs also significantly enhanced ALP activity induced by $\beta$-glycerophosphate and ascorbic acid. Furthermore, transfection of all the sFRP expression vectors significantly increased $\beta$-catenin/TCF reporter activity and the effects were most prominent with sFRP-2 and -4. In osteoblast apoptosis assay, only sFRP-3 increased etoposide-induced apoptosis in MC3T3-E1 mouse osteoblasts. In conclusion, we found that different repertoires of sFRPs exert differential effects on osteoblastic differentiation of mouse mesenchymal cells and cellular apoptosis of mouse osteoblasts in vitro.
\end{abstract}

(C) 2007 Elsevier Inc. All rights reserved.

Keywords: Secreted frizzled-related proteins; Wnt; Osteoblastic differentiation; Apoptosis

Wnts are a family of secreted glycoproteins that regulate various normal and pathological developmental process of many organs including bone (for review see Ref. [1]). In the canonical Wnt/ $\beta$-catenin pathway, binding of Wnt proteins to the frizzled receptors activates the intracellular Dishevelled, which inhibits the kinase activity of a complex containing glycogen synthetase kinase-3, Axin, $\beta$-catenin, and other proteins [2]. Since these complex targets $\beta$-catenin for rapid degradation through phosphorylation, Wnt signaling allows the stabilization, and nuclear translocation of $\beta$-catenin. $\beta$-Catenin then binds to TCF/LEF family of transcription factors to regulate the expression of Wnt target genes [1].

\footnotetext{
* Corresponding author. Fax: +82 27653734.

E-mail address: csshin@snu.ac.kr (C.S. Shin).
}

Growing number of evidences suggest that Wnt signaling plays a critical role in the regulation of bone accrual during growth and the establishment of peak bone mass as well as bone remodeling. A point mutation $(\mathrm{G} 171 \mathrm{~V})$ at the $\beta$-propeller module of LRP5, a Wnt co-receptor, results in high bone mass phenotype, whereas targeted disruption of LRP5 in mice or inactivating mutation in human leads to decreased bone formation [3]. Wnt5a is required for longitudinal skeletal outgrowth and both Wnt5a and Wnt5b regulate the transition between different chondrocyte zones [4].

Activity of Wnt signaling pathway is regulated by secreted inhibitors, including secreted frizzled-related protein (sFRP) [5], Wnt inhibitory factor-1 (WIF-1) [6], and Dickkopf (Dkk) [7]. The sFRP family has an amino-terminal cysteine-rich domain (CRD) that has high homology with the ligand-binding domain of frizzled Wnt receptor 
[8]. sFRPs bind directly to the Wnts proteins and appear to antagonize the effects of Wnts by sequestering them from their receptor. The inhibitory role of sFRPs on Wnt signaling in mesenchymal differentiation was supported by the finding that treatment of 3T3-L1 preadipocytes with sFRP-1 or sFRP-2 resulted in spontaneous differentiation to adipocytes in contrast to the inhibitory role of Wnt in adipogenesis [9]. However, the role of sFRPs in osteoblasts is still elusive. Although overexpression of sFRP-1 in human osteoblasts has been shown to antagonize Wnt signaling and accelerates apoptosis [10], a recent report demonstrated that SFRP-3 is a potent stimulator of osteoblast differentiation [11].

This study was undertaken for definitive assessment of contribution of a series of sFRPs on osteoblastic differentiation from mesenchymal progenitors using a murine mesenchymal C3H10T1/2 cell line. We have also investigated the effects of sFRPs on the cellular apoptosis of MC3T3E1, a mouse calvaria-derived osteoblastic cell line.

\section{Materials and methods}

Materials. Recombinant human sFRP-1, 3, 4, Dkk-1, and mouse sFRP-2 were purchased from R\&D system (Minneapolis, MN). Monoclonal anti-HA antibody (MMS 101-P) was obtained from Covance Inc. (Princeton, NJ). TOPflash plasmid which contains eight copies of the optimal Tcf motif CCTTTGATC upstream of a minimal thymidine kinase promoter driving luciferase expression and FOPflash plasmid that contains a mutated motif CCTTTGGCC of the Tcf-Lef-sensitive elements were obtained from Dr. Roberto Civitellli (Washington University, St. Louis). Western blotting detection reagents and $\left[\alpha-{ }^{32} \mathrm{P}\right] \mathrm{dCTP}$ from Amersham Int. (Buckinghamshire, UK). Random priming kits and reagents for the luciferase assay were purchased from Promega Corp. (Madison, WI) and Lipofectamine Plus from Invitrogen Corp. (Carlsbad, CA). Oligonucleotides were synthesized by Bioneer Corp. (Chungwon, Korea), and unless otherwise indicated, all other chemicals, including tissue culture medium, were from Sigma-Aldrich Chemical company (St. Louis, MO).

Cell culture. Murine mesenchymal cells C3H10T1/2 (American Type Culture Collection, Manassas, VA) are pluripotent cells and were grown in Dulbecco's Modified Eagle Medium (DMEM) containing 10\% FBS. During osteoblastic differentiation studies, C3H10T1/2 cells were cultured in DMEM with $10 \%$ FBS supplemented with either Wnt-3A conditioned medium or $50 \mu \mathrm{g} / \mathrm{ml}$ ascorbic acid and $10 \mathrm{mM} \beta$-glycerophosphate. The mouse MC3T3-E1 osteoblastic cells (RIKEN cell bank, Tsukuba, Japan) were derived from spontaneously immortalized calvaria cells [12] and maintained in DMEM medium with 10\% FBS. The 3T3-L1 pre-adipocytic cell line was a kind gift from Dr. Jae Bum Kim (Seoul National University, Seoul, Korea) and maintained in an immature state by culturing in DMEM supplemented with $20 \%$ FBS and $2.0 \mathrm{mM}$ glutamine. Normal mouse osteoblasts were isolated from 21-day-old fetal mouse calvariae using a well-characterized technique essentially as described previously [13].

Preparation of Wnt-3A conditioned media (CM). L Wnt-3A [14] cells, that stably secrete biologically active mouse Wnt-3A were grown in DMEM supplemented with $10 \% \mathrm{FBS}, 4 \mathrm{mM}$ L-glutamine supplemented with $0.4 \mathrm{mg} / \mathrm{ml} \mathrm{G}-418$. Upon reaching $80 \%$ confluence, cells were washed once with PBS and transferred into DMEM containing $2 \%$ FBS, $1 \times$ penicillin/streptomycin, and $4 \mathrm{mM}$ L-glutamine. Conditioned media (CM) was collected 1-day past confluence. We refer to the concentration of this media to be $1 \times$. The concentration of active Wnt-3A in the $1 \times$ Wnt-3A $\mathrm{CM}$ is estimated to be $5 \mathrm{nM}$ [15].

Retroviral vector construction. The mouse sFRP-1, 2, 3, and 4 cDNAs were obtained from Dr. Jeremy Nathans (Johns Hopkins University,
Baltimore, MD). The mouse Dkk-1 cDNAs was a kind gift from Dr. Christof Niehrs (Deutsches Krebsforschungszentrum, Heidelberg, Germany). A hemagglutinin (HA) epitope was incorporated at the C-terminal of sFRPs using PCR, then ligated into the BglII/EcoRI site of the pMSCV-GFP plasmid (a kind gift from Dr. Neil A. Clipstone at the Northwestern University, Chicago, IL), upstream of internal ribosomal entry site (IRES), giving pMSCV-sFRP-1-4-HA-GFP, and pMSCV-Dkk1-HA-GFP. The nucleotide sequences of the amplified sFRP coding region were confirmed by sequencing.

Retrovirus production and transduction of cell lines. To generate retroviral particle, 293T cells were transfected with DNA (4 $\mu \mathrm{g}$ pMD-gag-pol, $4 \mu \mathrm{g}$ pMD-VSVG, and $4 \mu \mathrm{g}$ retroviral vector pMSCV-sFRP-1-4-HAGFP, pMSCV-Dkk-1-HA-GFP, or pMSCV-GFP alone), lipofectamine PLUS, and serum-free DMEM. Viral supernatant fractions were collected at $48 \mathrm{~h}$ and transduced into $\mathrm{C} 3 \mathrm{H} 10 \mathrm{~T} 1 / 2$ cells as described previously [16]. Transduced cells expressing higher level of GFP were sorted using FACS (FACSVantage SE, Beckton-Dickinson) and used for studies.

Alkaline phosphatase (ALP) activity. To assess ALP activities, cells were washed three times with ice-cold Tris-buffered saline (TBS), pH 7.4 and scraped immediately after adding $0.5 \mathrm{~mL}$ of ice-cold $50 \mathrm{mM}$ TBS; the collected lysates were then sonicated for $20 \mathrm{~s}$ at $4{ }^{\circ} \mathrm{C}$. Enzyme activity assay was performed in assay buffer $\left(10 \mathrm{mM} \mathrm{MgCl}_{2}\right.$ and $0.1 \mathrm{M}$ alkaline buffer, $\mathrm{pH} 10.3$ ) containing $10 \mathrm{mM}$-nitrophenylphosphate as substrate. The reaction was stopped by adding $0.3 \mathrm{~N} \mathrm{NaOH}$ absorbance was read at $\mathrm{OD}_{405}$. Relative ALP activity is defined as mmol of $p$-nitrophenol phosphate hydrolyzed per min per $\mathrm{mg}$ of total protein.

$R T$-PCR. First-strand cDNA was synthesized from $1 \mu \mathrm{g}$ of total RNA using a Reverse Transcription System kit (Promega, Madison, WI). PCR was performed using $100 \mathrm{ng}$ of cDNA, $20 \mathrm{pmol}$ of each primer (synthesized by Bioneer Corp., Chungwon, Korea), $2.5 \mathrm{mM}$ of dNTPs, $1 \mathrm{mM}$ of $\mathrm{MgCl}_{2}$, and $1 \mathrm{U}$ of Taq polymerase in a $50 \mu \mathrm{l}$ reaction volume containing $1 \times$ Taq polymerase buffer using a Perkin-Elmer Gene Amp PCR System 9600. The forward and reverse primer sequences used to amplify sFRPs and $\beta$-actin are listed in Supplementary Table 1.

Transfections and reporter assays. For these experiments, C3H10T1/2 cells were seeded into 24 -well plates at 50,000 cells/well. Twenty-four hours later, TOPflash or FOPflash plasmid was cotransfected with expression vectors for sFRP-1-4 or Dkk-1 in the presence or absence of Wnt-3A CM. Forty-eight hours later, cell lysates were prepared using the Promega Luciferase assay system, and activity was measured using a luminometer (Lumat LB 9507, Berthold, Germany). All the transient transfections were performed in triplicate and all luciferase values were normalized against the $\beta$-galactosidase activities from the cotransfected pCMV- $\beta$-gal plasmid. All values, means and standard deviations were expressed relative to basal promoter activity as a fold induction.

Western blot analysis. Cells were lysed in $50 \mathrm{mM}$ Tris $(\mathrm{pH} 7.5)$, $150 \mathrm{mM} \mathrm{NaCl}$, and $1 \%$ Triton X-100 supplemented with a protease inhibitor mixture (Sigma, added at a dilution of 1:100). Lysates were separated by SDS-PAGE, and transferred to a Hybond ECL nitrocellulose membrane (Amersham, Piscataway, NJ). The membrane was first blocked with $5 \%$ milk for $1 \mathrm{~h}$, and then incubated with primary and secondary antibodies for 24 and $1 \mathrm{~h}$, respectively. Development of the membrane was performed using an enhanced chemiluminescence kit (Amersham, Piscataway, NJ).

Quantification of apoptosis. MC3T3-E1 cells were cultured in serumstarved condition for $24 \mathrm{~h}$, and sequentially treated with $20 \mathrm{nM}$ of sFRP-1, 2, 3, or 4 for $1 \mathrm{~h}$. Apoptosis was induced by the addition of etoposide $\left(10^{-3} \mathrm{M}\right)$ (Sigma-Aldrich Chemical company, St. Louis, MO) for $10 \mathrm{~h}$ and the extent of apoptosis was quantified by assessing the characteristic nuclear, i.e., chromatin condensation and nuclear fragmentation, by using the 4',6-diamidino-2-phenylindole dihydrochloride (DAPI, Sigma-Aldrich, St. Louis, MO) staining under the fluorescence microscope.

Statistical analysis. All data are presented as means \pm SD. The data were analyzed by one-way ANOVA. When the ANOVA indicated a significant difference among the groups, statistical differences between two groups were evaluated by Student-Newman-Keuls multiple comparison test. A $p$ value $<0.05$ was considered significant for all statistical analyses. 


\section{Results}

\section{Expression of sFRPs in murine cells of mesenchymal origin}

We first investigated the endogenous expression of sFRPs in a number of murine cells of mesenchymal origin by RT-PCR analysis; pluripotent mesenchymal C3H10T1/ 2, osteoblastic MC3T3-E1, myoblastic C2C12, pre-adipocytic 3T3-L1, and primary cultures of mouse calvaria. Expected cDNA fragments corresponding to mouse sFRP-1, 2, 3, 4 were clearly amplified from RNA of these cells (Fig. 1A). The expression level of sFRP-1 was the highest in MC3T3-E1 cells whereas both sFRP-2 and -4 were most highly expressed in $\mathrm{C} 2 \mathrm{C} 12$ cell compared to other cell types. The expression of sFRP-3 was comparable in $\mathrm{C} 2 \mathrm{C} 12, \mathrm{MC} 3 \mathrm{~T} 3-\mathrm{E} 1$, and $\mathrm{C} 3 \mathrm{H} 10 \mathrm{~T} 1 / 2$ cells.
Effects of sFRP treatment on osteoblastic differentiation of C3H10T1/2 cells

When $\mathrm{C} 3 \mathrm{H} 10 \mathrm{~T} 1 / 2$ cells were treated with $0.5 \times$ Wnt-3A conditioned media (CM) for $72 \mathrm{~h}$, the ALP activity was increased by 10- to 25-folds (Fig. 1B, Wnt-3A) as expected. Co-treatment with 5-100 nM of sFRP-1, 2, 3, or 4 in this setting, however, did not significantly inhibit the ALP activity (Fig. 1B). Actually, sFRP-2 at concentrations of 10,50 , and $100 \mathrm{nM}$ (Fig. 1B-b) and sFRP-4 at low concentrations (5 nM, Fig. 1B-d) significantly increased ALP activities. The effects of sFRPs on ALP activity did not seem to have any dose-responsive pattern. In contrast, in a parallel experiment, the treatment with Dkk-1, a robust inhibitor of Wnt signaling, has consistently inhibited the ALP activity in a dose-responsive manner (Fig. 1B-e).

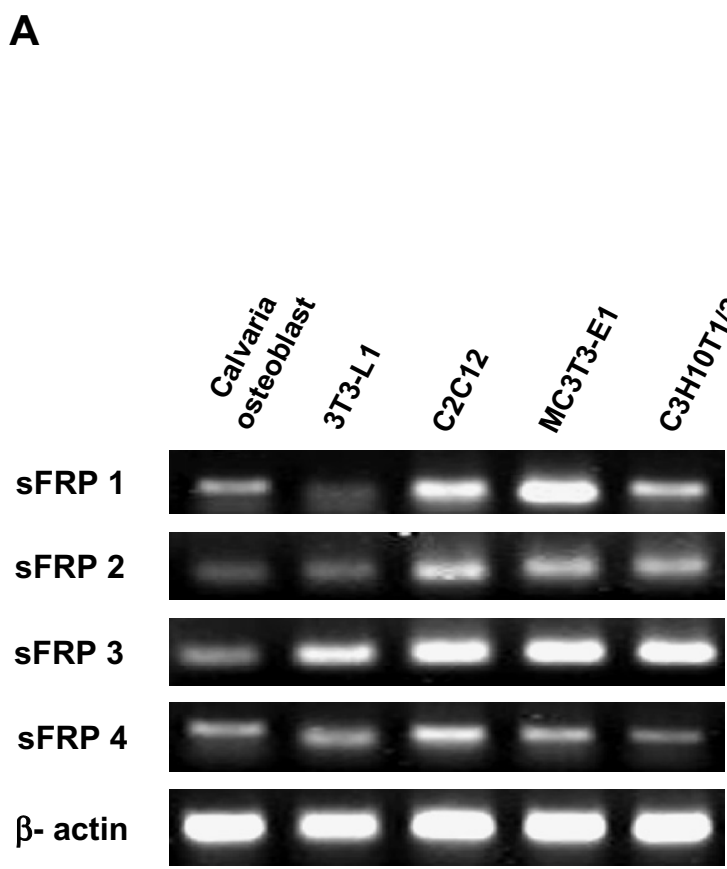

B a
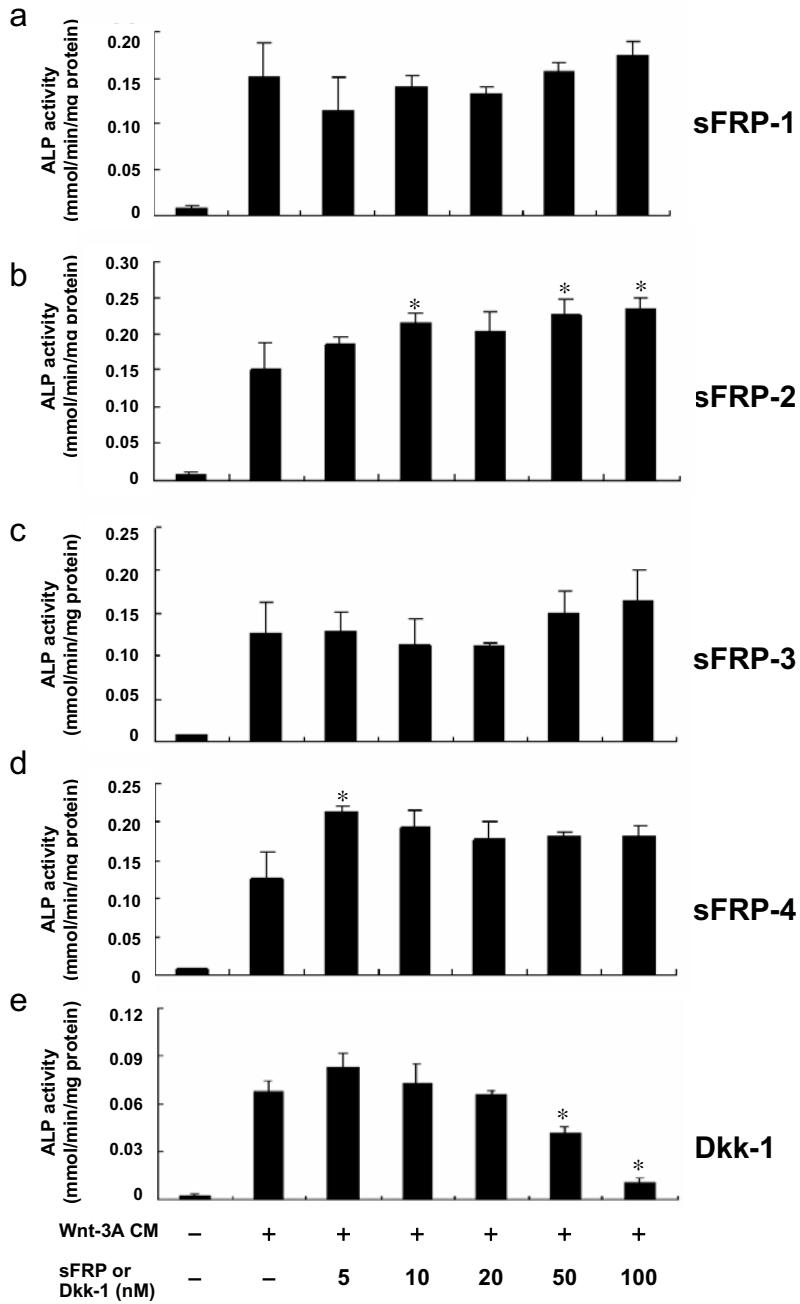

Fig. 1. Expression of sFRPs in mesenchymal cells and effect of sFRPs on the ALP activities induced by Wnt-3A in C3H10T1/2 cells. (A) RT-PCR analysis for sFRP-1, 2, 3, 4, and $\beta$-actin were performed from reverse-transcribed total RNA prepared from primary culture of calvarial osteoblasts, pre-adipocytic 3T3-L1 cells, myoblastic C2C12 cells, osteoblastic MC3T3-E1 cells, and mouse mesenchymal C3H10T1/2 cells. (B) C3H10T1/2 cells were seeded in 24-well plate and grown for $72 \mathrm{~h}$ in DMEM containing $10 \%$ FBS and treated with vehicle or varying concentrations of sFRP-1-4 or Dkk-1 in the presence or absence of Wnt-3A. ALP activity was determined and normalized to protein content. The results are representative of three independent experiments performed in triplicate. Each bar represents means $\pm \mathrm{SD} .{ }^{*} p<0.05$ vs. vehicle. 
Effects of retrovirally transduced sFRPs on osteoblastic differentiation of C3H1OT1/2 Cells

To further verify the role of sFRPs in the osteoblastic differentiation of mesenchymal cells, we studied the effects of the sFRPs treatment during cultures in osteogenic medium, i.e., $\beta$-glycerophosphate and ascorbic acid, a more physiologic stimulator of osteoblastic differentiation. We used stable cell lines that endogenously overexpressed sFRPs in C3H10T1/2 cells using retrovirus. In this model, the overexpressed sFRPs are expected to work by autocrine and/or paracrine mechanism. The expression of sFRPs were examined by Western blot analysis using an antibody directed against the HA epitope (Fig. 2A).

As expected, culture of $\mathrm{C} 3 \mathrm{H} 10 \mathrm{~T} 1 / 2$ cells transduced with empty virus (MSCV) in osteogenic medium for 14 days exhibited typical induction of ALP activity (Fig. 2B). Overexpression of sFRP-2 in this condition resulted in significant increase in the ALP activity on day 14 of culture, whereas other sFRPs did not. These results are analogous with the data we obtained from the exogenous treatment of the cells with sFRPs in the presence of Wnt-3A CM and suggest that the effects of sFRPs are not necessarily negative in the regulation of osteoblastic differentiation of mesenchymal cell line.

\section{Effect of sFRPs on Wnt- $\beta$-catenin transcriptional activity}

We next examined the effects of sFRPs on the $\beta$-catenin/ Tcf transcriptional activity, a downstream target of the Wnt signaling. Transient transfection of TOPflash in C3H10T1/2 cells showed only a basal level of transactivation, and this activity was increased by 8 -fold with the treatment with Wnt-3A CM (Fig. 3). Co-transfection with sFRP-1, -2, -3, or sFRP-4 expression plasmid in this setting resulted in significant increase in the transcriptional activity. Among the sFRPs, sFRP-2 and -4 exhibited most strong responses. As a control, Dkk-1 significantly inhibited the reporter activity as expected. Same experiments performed using the FOPflash reporter did not reveal any changes regardless of treatment with Wnt-3A.

\section{Effect of sFRPs on apoptosis of osteoblastic MC3T3-E1 cells}

Since none of the sFRPs has negatively regulated osteoblastic differentiation of mesenchymal C3H10T1/2 cells, we next evaluated whether sFRPs increases apoptosis of osteoblasts thereby contributing to net loss of bone as has been reported in sFRP-1 transgenic model [17]. Towards this end, we treated topoisomerase inhibitor etoposide to MC3T3-E1 cells after $1 \mathrm{~h}$ pre-conditioning with sFRPs or
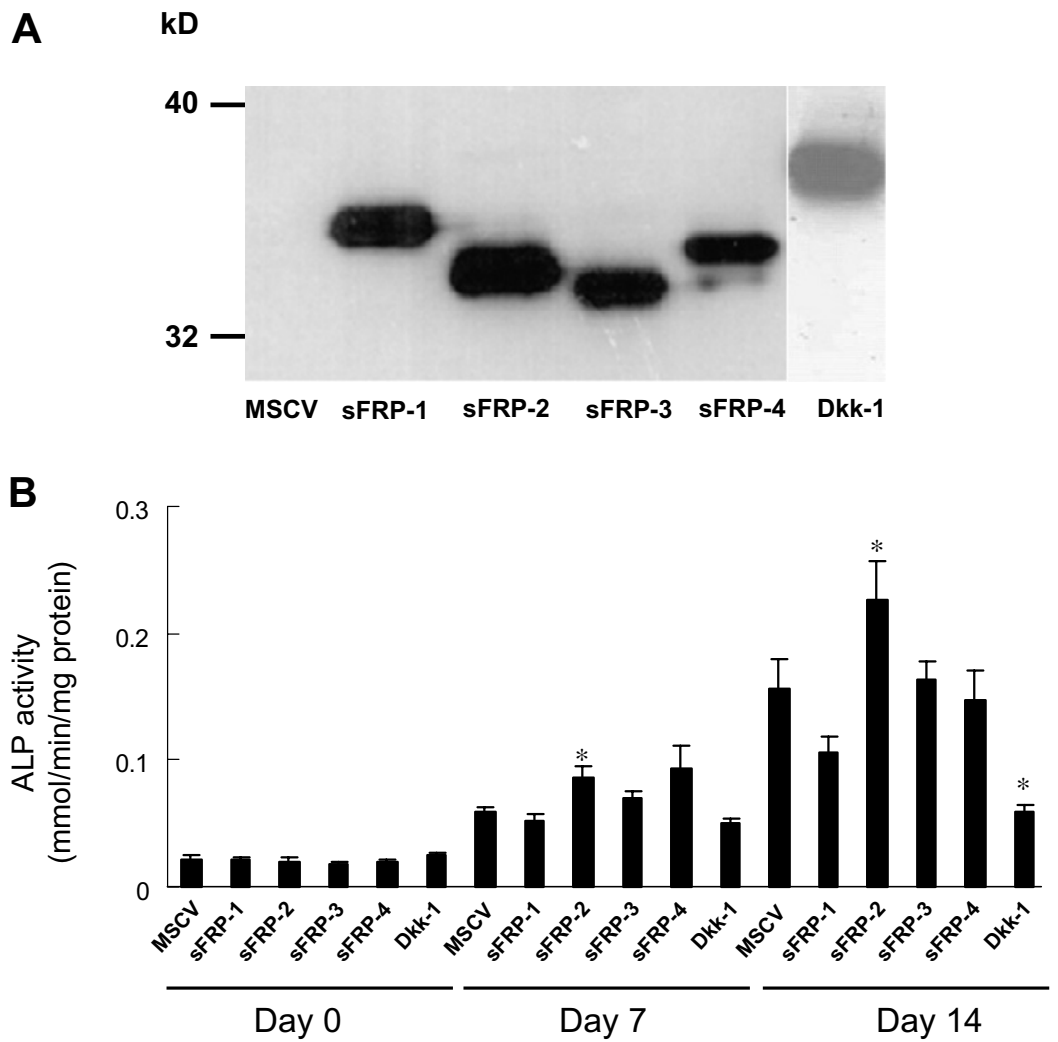

Fig. 2. Effect of sFRP retrovirus on the ALP activities induced by osteogenic medium. (A) C3H10T1/2 cells transduced with sFRP-1, 2, 3, 4, or Dkk-1 retrovirus. Western blot analyses were performed using antibodies against HA epitope. (B) C3H10T1/2 cells stably transduced with sFRP-1, 2, 3, 4, or Dkk-1 retrovirus were seeded in 24-well plate and grown in DMEM containing 10\% FBS in the presence or absence of $\beta$-glycerophosphate and ascorbic acid for 14 days. ALP activity was determined and normalized to protein content. The results are representative of three independent experiments performed in triplicate. Each bar represents means $\pm \mathrm{SD} .{ }^{*} p<0.05$ vs. vehicle. 


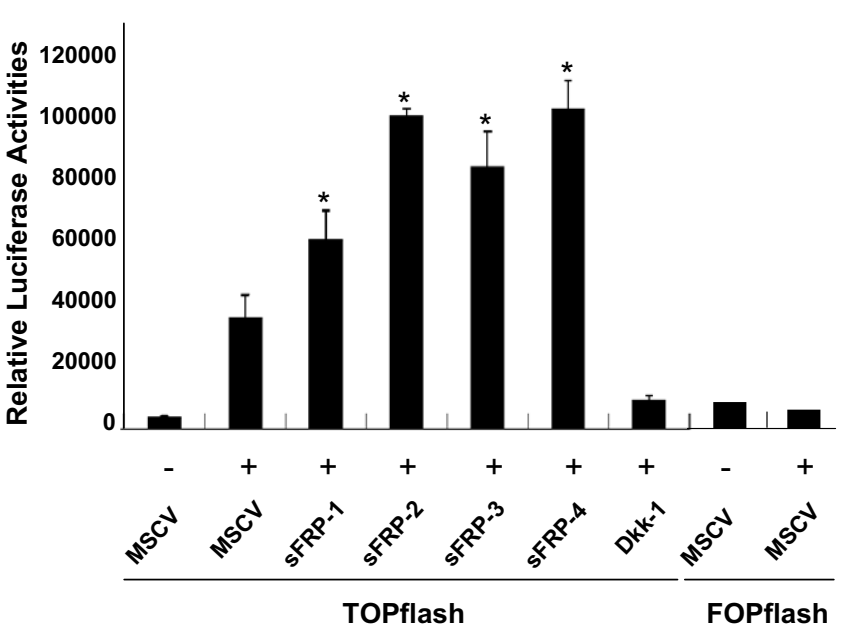

Fig. 3. Effect of sFRPs on the TOPflash activities induced by Wnt-3A in C3H10T1/2 cells. C3H10T1/2 cells were co-transfected with sFRP-1, 2, 3, 4, or Dkk-1 expression vectors and TOPflash or FOPflash plasmid. Fortyeight hours after transfection, luciferase activity was measured and normalized for transfection efficiency against $\beta$-galactosidase activity from the co-transfected pCMV- $\beta$-gal plasmid. Values are presented as means $\pm \mathrm{SD}$, and results are representative of three experiments each performed in triplicate. Each bar represents means $\pm \mathrm{SD} .{ }^{*} p<0.05$ vs. MSCV.

Dkk-1. Quantification of apoptotic cells with DAPI staining showed that only sFRP-3 significantly increased etoposide-induced apoptosis in mouse osteoblastic cells (Fig. 4), whereas other subtypes did not affect cell death.

\section{Discussion}

In this study we have shown that the major secretary Wnt modulators, sFRPs, including sFRP-1, 2, 3, and 4, did not inhibit the Wnt-3A-mediated increase in ALP activities in C3H10T1/2 mesenchymal cells in vitro. On the contrary, both sFRP-2 and sFRP-4 have actually increased ALP activities.
We have found that all the mesenchymal cell lines express varying degree of sFRP expression. These results initially suggested that these molecules may serve physiologic functions in these cells as an auto- or paracrine modulators of Wnt signaling. We hypothesized that sFRPs, by binding directly to the Wnts proteins and disrupting of $\mathrm{Wnt} / \beta$-catenin signaling, would inhibit the osteoblastic differentiation. However, in our study we were unable to identify the negative roles of sFRPs in the ALP activity induced by Wnt-3A in C3H10T1/2 cell line. Indeed, both sFRP-2 and -4 have significantly enhanced ALP activity at low concentrations, and at higher concentrations $(>=50 \mathrm{nM})$, sFRP-2 has consistently increased ALP activities. This lack of inhibitory effects by all the sFRPs was also reproduced when we transduced the cells with retrovirus rendering the cells to produce sFRPs endogenously. This approach was employed because sFRPs tend to remain predominantly associated with the cell membrane or extracellular matrix [18]. In addition, transduction of sFRP-2 retrovirus has also resulted in increase in ALP activities compared to control virus. Therefore, the results from the retroviral transduction study further confirm that even endogenously secreted sFRPs that should have autocrine or paracrine effects, do not inhibit the osteoblastic differentiation capabilities in these $\mathrm{C} 3 \mathrm{H} 10 \mathrm{~T} 1 / 2$ cells.

The lack of inhibition and even enhancement of ALP activity by sFRPs (sFRP-2 and -4) in our experiments is an unexpected result. The reason for this discrepancy with previous reports is currently unknown. One explanation might be that the sFRPs may have differential effects depending on the concentration. Indeed, Uren and his co-worker showed that sFRP-1 reduces Wingless $(\mathrm{Wg}$, Drosophila homolog of Wnt) activity only at high concentrations around 300-600 $\mathrm{nM}[19]$ whereas at low concentrations it potentiates $\mathrm{Wg}$ activity in Drosophila S2 cells [19]. Moreover, Chung et al. also showed that sFRP-3 at a concentration of $10 \mathrm{nM}$ increased Wnt-3A-mediated increase in ALP activity [11]. Alternatively, the sFRPs

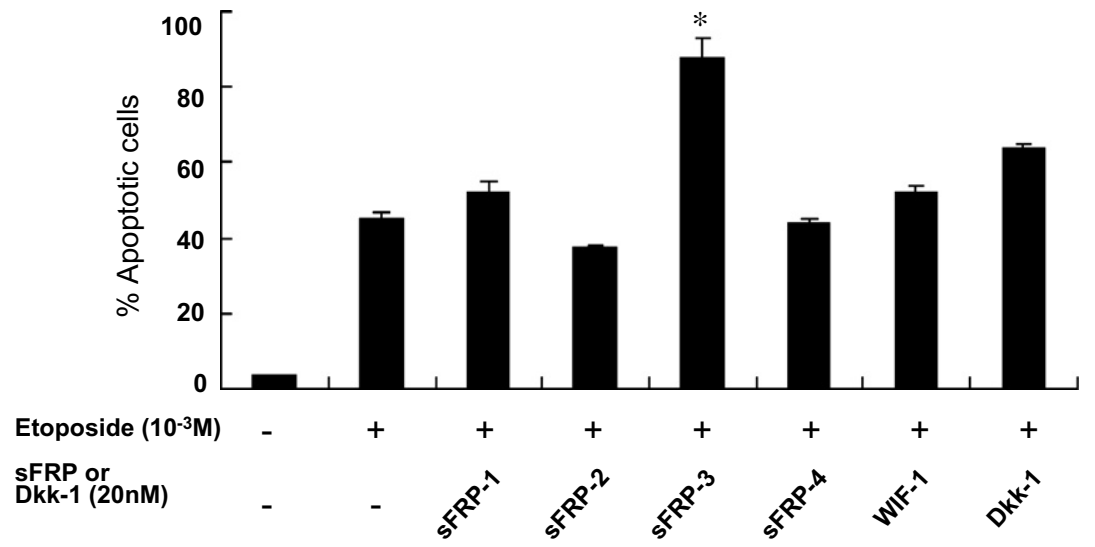

Fig. 4. Effect of sFRPs on apoptosis in MC3T3-E1 cells. After $24 \mathrm{~h}$ serum-starved culture, MC3T3-E1 cells were pretreated with $20 \mathrm{nM}$ of sFRP-1, 2, 3, 4, and Dkk-1 for $1 \mathrm{~h}$ and then $10^{-3} \mathrm{M}$ of etoposide was added. After $24 \mathrm{~h}$, apoptosis was quantified by $4^{\prime}, 6$-diamidino-2-phenylindole dihydrochloride (DAPI) staining and fluorescence microscopy. The results are representative of three independent experiments performed in triplicate. Each bar represents means \pm SD. ${ }^{*} p<0.05$ vs. vehicle. 
may have stage-specific effects on the osteoblast differentiation with little or no effects on undifferentiated mesenchymal stage but significant effects on pre-osteoblasts or mature osteoblasts in vitro [17]. Lastly, there may be some specificity in interactions between particular sFRPs and Wnt molecules so that the sFRPs are not capable to specifically inhibit the action of Wnt-3A. In support of this notion, Wang et al. has demonstrated that sFRP-3 (Frzb1) is not able to inhibit the action of Wnt-3A or $-5 \mathrm{~A}$, whereas it inhibits Wnt-1 or -8 [20], suggesting that sFRPs modulates Wnt activity depending on the subtypes of both Wnt and sFRPs.

We have also shown that all the sFRPs increased TOPflash activity induced by Wnt-3A. Moreover, the prominent effects by sFRP-2 and -4 suggest that the increase in ALP activities by both of sFRPs may result from the increased $\beta$-catenin/Tcf transcriptional activities. However, although ALP is one of the downstream targets of Wnt signaling [21], we have not further dissected molecular mechanisms whereby sFRPs exerted ALP activities and osteoblastic differentiation. Additional studies on the ligand and receptor-binding activities and the events downstream of frizzled receptors could help answer this question.

Since sFRPs did not inhibit osteoblastic differentiation of mesenchymal progenitors, we next reasoned that these secretary modulators may promote apoptosis of mature osteoblasts. This is based on the previous in vivo demonstration of increased apoptosis of osteoblasts in sFRP-1 overexpressing mice [17]. However, unlike the animal model, only sFRP-3 promoted apoptosis induced by etoposide in our experiment. Although differences in the experimental model preclude direct comparison, these results also indicate that there are a lot of disparities in the action of sFRPs depending on the study model and subtypes.

In conclusion, we have shown that different repertoires of sFRPs have differential effects on osteoblastic differentiation and TCF/LEF transcriptional activities in murine mesenchymal cells and also on the apoptosis of osteoblasts. We speculate that the contradictory effects of sFRPs result from difference in interactions between different sFRPs and Wnts, diverse repertoires of Wnt molecules, and tissue-specific or stage-specific responses, as well as biphasic response depending on the concentrations of sFRPs.

\section{Acknowledgment}

This work was supported by a grant from Ministry of Health and Welfare of Korea (Grant No. A060371).

\section{Appendix A. Supplementary data}

Supplementary data associated with this article can be found, in the online version, at doi:10.1016/j.bbrc.2007. 12.128 .

\section{References}

[1] K.M. Cadigan, R. Nusse, Wnt signaling: a common theme in animal development, Genes Dev. 11 (1997) 3286-3305.

[2] E. Hay, C. Faucheu, I. Suc-Royer, R. Touitou, V. Stiot, B. Vayssiere, R. Baron, S. Roman-Roman, G. Rawadi, Interaction between LRP5 and Frat 1 mediates the activation of the Wnt canonical pathway, J. Biol. Chem. 280 (2005) 13616-13623.

[3] R. Baron, G. Rawadi, S. Roman-Roman, Wnt signaling: a key regulator of bone mass, Curr. Top. Dev. Biol. 76 (2006) 103-127.

[4] Y. Yang, L. Topol, H. Lee, J. Wu, Wnt5a and Wnt5b exhibit distinct activities in coordinating chondrocyte proliferation and differentiation, Development 130 (2003) 1003-1015.

[5] Y. Kawano, R. Kypta, Secreted antagonists of the Wnt signalling pathway, J. Cell Sci. 116 (2003) 2627-2634.

[6] J.C. Hsieh, L. Kodjabachian, M.L. Rebbert, A. Rattner, P.M. Smallwood, C.H. Samos, R. Nusse, I.B. Dawid, J. Nathans, A new secreted protein that binds to Wnt proteins and inhibits their activities, Nature 398 (1999) 431-436.

[7] B. Mao, W. Wu, G. Davidson, J. Marhold, M. Li, B.M. Mechler, H. Delius, D. Hoppe, P. Stannek, C. Walter, A. Glinka, C. Niehrs, Kremen proteins are Dickkopf receptors that regulate Wnt/betacatenin signalling, Nature 417 (2002) 664-667.

[8] A. Wodarz, R. Nusse, Mechanisms of Wnt signaling in development, Annu. Rev. Cell Dev. Biol. 14 (1998) 59-88.

[9] C.N. Bennett, S.E. Ross, K.A. Longo, L. Bajnok, N. Hemati, K.W. Johnson, S.D. Harrison, O.A. MacDougald, Regulation of Wnt signaling during adipogenesis, J. Biol. Chem. 277 (2002) 30998-31004.

[10] P.V. Bodine, J. Billiard, R.A. Moran, H. Ponce-de-Leon, S. McLarney, A. Mangine, M.J. Scrimo, R.A. Bhat, B. Stauffer, J. Green, G.S. Stein, J.B. Lian, B.S. Komm, The Wnt antagonist secreted frizzledrelated protein-1 controls osteoblast and osteocyte apoptosis, J. Cell. Biochem. 96 (2005) 1212-1230.

[11] Y.S. Chung, D.J. Baylink, A.K. Srivastava, Y. Amaar, B. Tapia, Y. Kasukawa, S. Mohan, Effects of secreted frizzled-related protein 3 on osteoblasts in vitro, J. Bone Miner. Res. 19 (2004) 1395-1402.

[12] H. Sudo, H.A. Kodama, Y. Amagai, S. Yamamoto, S. Kasai, In vitro differentiation and calcification in a new clonal osteogenic cell line derived from newborn mouse calvaria, J. Cell Biol. 96 (1983) 191-198.

[13] F. Furlan, F. Lecanda, J. Screen, R. Civitelli, Proliferation, differentiation and apoptosis in connexin43-null osteoblasts, Cell Commun. Adhes. 8 (2001) 367-371.

[14] S. Shibamoto, K. Higano, R. Takada, F. Ito, M. Takeichi, S. Takada, Cytoskeletal reorganization by soluble Wnt-3a protein signalling, Genes Cells 3 (1998) 659-670.

[15] L.M. Galli, T. Barnes, T. Cheng, L. Acosta, A. Anglade, K. Willert, R. Nusse, L.W. Burrus, Differential inhibition of Wnt-3a by Sfrp-1, Sfrp-2, and Sfrp-3, Dev. Dyn. 235 (2006) 681-690.

[16] C.S. Shin, S.J. Her, J.A. Kim, H. Kim do, S.W. Kim, S.Y. Kim, H.S Kim, K.H. Park, J.G. Kim, R. Kitazawa, S.L. Cheng, R. Civitelli, Dominant negative $\mathrm{N}$-cadherin inhibits osteoclast differentiation by interfering with beta-catenin regulation of RANKL, independent of cell-cell adhesion, J. Bone Miner. Res. 20 (2005) 2200-2212.

[17] P.V. Bodine, W. Zhao, Y.P. Kharode, F.J. Bex, A.J. Lambert, M.B Goad, T. Gaur, G.S. Stein, J.B. Lian, B.S. Komm, The Wnt antagonist secreted frizzled-related protein-1 is a negative regulator of trabecular bone formation in adult mice, Mol. Endocrinol. 18 (2004) $1222-1237$

[18] A. Bafico, A. Gazit, T. Pramila, P.W. Finch, A. Yaniv, S.A. Aaronson, Interaction of frizzled related protein (FRP) with Wnt ligands and the frizzled receptor suggests alternative mechanisms for FRP inhibition of Wnt signaling, J. Biol. Chem. 274 (1999) 1618016187.

[19] A. Uren, F. Reichsman, V. Anest, W.G. Taylor, K. Muraiso, D.P. Bottaro, S. Cumberledge, J.S. Rubin, Secreted frizzled-related protein-1 binds directly to Wingless and is a biphasic modulator of Wnt signaling, J. Biol. Chem. 275 (2000) 4374-4382. 
[20] Y.K. Wang, C.H. Samos, R. Peoples, L.A. Perez-Jurado, R. Nusse, U. Francke, A novel human homologue of the Drosophila frizzled Wnt receptor gene binds wingless protein and is in the Williams syndrome deletion at 7q11.23, Hum. Mol. Genet. 6 (1997) 465-472.
[21] G. Rawadi, B. Vayssiere, F. Dunn, R. Baron, S. Roman-Roman, BMP-2 controls alkaline phosphatase expression and osteoblast mineralization by a Wnt autocrine loop, J. Bone Miner. Res. 18 (2003) 1842-1853. 